\title{
Physiological responses of Escherichia coli cells cultivated under a sublethal oxidative stress condition
}

\author{
Krishnaiah, D., ${ }^{1 *}$ Bono, A., ${ }^{1}$ Sarbatly, R., ${ }^{1}$ \\ Nishioka, M., ${ }^{2}$ Kim, S. Y., ${ }^{2}$ Tanaka, M., ${ }^{2}$ Ojima, Y. ${ }^{2}$ and Taya, ${ }^{2}$ \\ ${ }^{1}$ Chemical Engineering Programme, School of Engineering and Information Technology \\ Universiti Malaysia Sabah, 88999 Kota Kinabalu, Sabah \\ ${ }^{2}$ Division of Chemical Engineering, Department of Materials Engineering Science, Graduate School of Engineering Science, \\ Osaka University, Toyonaka, 560-8531, Osaka, Japan \\ E-mail: krishna@ums.edu.my
}

\begin{abstract}
Superoxide dismutase (SOD) is widely distributed in organisms and alleviates toxicity of reactive oxygen species (ROS) formed inside cells. It was found that sublethal oxidative stress derived from photoexcited $\mathrm{TiO}_{2}$ exerted a simulative effect on the growth of SOD null mutant of Escherichia coli (IM303) with reduction in intracellular ROS level. DNA microarray analysis was then carried out to compare gene expression between IM303 cells with and without the oxidative stress. From the DNA micro array data, $y f i D, y g g B$ and $y g g E$ were selected as genes up- regulated under the oxidative condition and then cloned into a pUC 19 plasmid. The original pUC 19 and constructed plasmids were introduced into E. coli MM294 and the transformants were cultivated in M9 medium with paraquat. Among these transformants, intracellular ROS content was the lowest in the cells carrying yggE gene and maximum specific growth rate of those cells was also higher than that of control cells with pUC 19. These results suggest that $y g g E$ gene product has an ROS-scavenging function in the cells of $E$. coli cells exposed to an oxidative stress and improves the efficiency of cellular growth.
\end{abstract}

Keywords: Escherichia coli, reactive oxidative stress, cell cultivation, photoexcited $\mathrm{TiO}_{2}$.

\section{INTRODUCTION}

Oxidative stresses are generated by variety of mechanisms such as normal aerobic metabolism, exposure to ultraviolet light, metal ions, and oxidative components (Halliwell and Gutteridge, 1989, Canghai Lu et al., 2005, Stortz and Imlay, 1999, Pomposiello and Demple, 2000, Kren et al., 1988, Farr and Kogoma, 1991). The aerobic organisms have high reducing potential of molecular oxygen, and preferentially utilize oxygen for their vital function and proliferation (Inaoka et al., 1998). Consequently, reactive oxygen species (ROS) are often formed and can oxidize to damage various biomaterials owing to their high reactivity. Superoxide dismutase (SOD) is known as a key component in cellular defense against oxidative stress through reducing the intracellular concentration of superoxide anion to maintain cellular viability (Carlioz and Touati, 1986). It was demonstrated that E. coli mutants lacking in SOD displayed several defects in phenotypic features when the cells were exposed to an aerobic condition (Gort and Imlay, 1998).

During the last decade, important works report strong and fast photocatalytic cell inactivation of $E$. coli and different bacteriophages with $\mathrm{TiO}_{2}$ (Ireland et al.,1993,
Bekbolet, 1997, Armon et al., 1998) and the plasmid DNA molecules were damaged by the oxidative stress in the $\mathrm{TiO}_{2}$ photoreaction (Kim et al., 2004a). It was reported that SOD-deficient mutants of Escherichia coli exhibited several defects, such as auxotrophy for amino acids, and high frequencies of spontaneous mutagenesis when grown aerobically (Gort and Imlay, 1998, Touati, 2002). These results also suggested that a sublethal or moderate level of oxidative stress may exert a triggering effect on activation of specific signaling, resulting in induction of altered metabolisms and cellular properties in SOD-deficient $E$. coli cells. However, recently the authors found that the population of SOD-efficient mutant of $E$. coli $\mathrm{M} 303$ contained a trace of spontaneously derived variant cells which prevailed during the culture under sublethal oxidative stress generated by photoexcited $\mathrm{TiO}_{2}$ (Kim et al., 2004). This result suggested that the variant cells possibly acquired a certain defense system against the oxidative stress besides the SOD mechanism, and the isolates exhibited the outgrowing properties with increased specific growth values under both the conditions with, and without $\mathrm{TiO}_{2}$ and light.

In the present study, the biological function of genes selected from the analysis was investigated in

*Corresponding author 
terms of cell growth and ROS content exposed to the oxidative stress generated by photoexicited $\mathrm{TiO}_{2}$.

\section{MATERIALS AND METHODS}

\section{Strain, media, cell growth, and cell isolation}

A mutant of E. coli, SOD-deficient strain IM303 (I4), and its wild-type strain MM294 were used throughout the experiments. A modified M9 medium containing $8 \mathrm{~g} / \mathrm{L}$ glucose was used with supplementation of amino acid mixture (2068 mixture, ATCC culture medium). IM303 (14) cells were grown at $37{ }^{\circ} \mathrm{C}$ in an L-type test tube containing $10 \mathrm{~mL}$ of the medium with or without $10 \mathrm{mg} / \mathrm{L}$ $\mathrm{TiO}_{2}$ particles (P25, Nippon Aerosil Co., Japan) under light irradiation at $12.5 \mathrm{~W} / \mathrm{m}^{2}$ with $20 \mathrm{~W}$ black light fluorescent lamps. In the case of MM294 culture, the cells were grown with $10 \mu \mathrm{mol} / \mathrm{L}$ paraquat $(\mathrm{PQ})$ under the same condition in the absence of $\mathrm{TiO}_{2}$ and light. Growth of the cells was monitored by measuring an optical density at $660 \mathrm{~nm}\left(\mathrm{OD}_{660}\right)$. Maximum specific growth rate, $\mu_{\mathrm{m}}$, was estimated by fitting the obtained growth-curve data to the modified Gompertz equation (Zwietering et al., 1990). To investigate effects of the selected gene on growth property, a bioreactor with $3 \mathrm{~L}$ vessel equipped with dissolved oxygen concentration and $\mathrm{pH}$ sensors (BMS-03PI, ABLE \& Biott Co., Ltd., Japan) was used. Cultures of recombinant IM303 (I4) cells were conducted in the following condition: $1.0 \mathrm{~L}$ of modified M9 medium containing $50 \mu \mathrm{g} / \mathrm{mL}$ ampicillin and $10 \mu \mathrm{mol} / \mathrm{L}$ isopropyl$\beta-D$-thiogalactopyran oside, $1 \%$ inoculation, $1.0 \mathrm{~L} / \mathrm{min}$ $(1.0 \mathrm{v} / \mathrm{v} / \mathrm{m})$ aeration, and $500 \mathrm{rpm}$ of agitation. Amount of cells was measured by monitoring $\mathrm{OD}_{660}$, and converted into dry cell weight. Glucose concentration in the medium was measured (Kim et al., 2005) by using an analytical instrument (BioProfile 200 Yamato Scientific Co., Ltd., Japan).

\section{Irradiation experimental setup}

The experimental setup is shown schematically in Figure 1. All the experiments were carried out in L-type test tube containing $10 \mathrm{~mL}$ of medium with or without $0.01 \mathrm{~g} / \mathrm{dm}^{3}$ $\mathrm{TiO}_{2}$ particles (Degussa P25, Japan Aerosil Co., Japan) under shaking condition of 45 strokes per minute. The test tubes were irradiated from the upper side with a bank of $20 \mathrm{~W}$ black light fluorescent lamps with effective wavelengths of $300-420 \mathrm{~nm}$ (FL-20S BL-B, Matsushita Electric Industrial Co., Japan). The incident light intensity was adjusted by changing the vertical distance between the lamps and test tubes, and was recorded as an average measurements conducted on the tube surfaces with a quantum sensor (Model No. J-221, UVP Inc., USA).

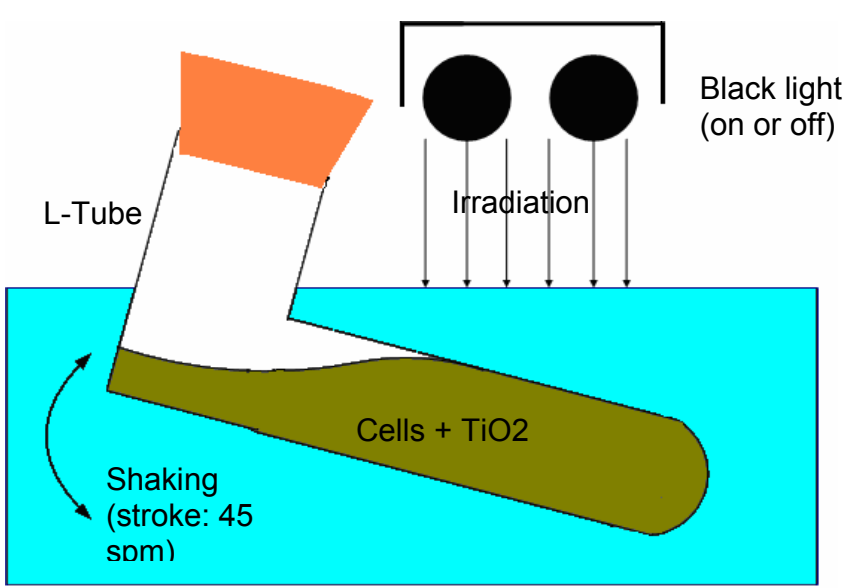

Figure 1: Experimental setup for cultivating E.coli strain under oxidative condition

DNA micro array analysis and construction of plasmid carrying a selected gene

IM303 (14) cells cultivated with/without light-irradiated $\mathrm{TiO}_{2}$ were harvested from the culture solution, and total RNA was extracted from the whole cells by employing a RNeasy Mini Kit and an RNase-free DNase Set (QIAGEN Inc., USA). Labeled cDNAs were prepared using a RNA Fluorescence Labeling Core Kit (Takara Bio Inc., Japan) with dUTP-Cy3 or dUTP-Cy5 fluorescent nucleotide. Assay of DNA microarray was conducted with an IntelliGene ${ }^{\circledR}$ E. coli CHIP (Takara Bio Inc., Japan). The microarray profiling data were compared between RNA samples from IM303 (14) cultures with, and without the oxidative stress was generated by photoexcited $\mathrm{TiO}_{2}$. The constitutive regions of the selected genes were amplified by PCR. A pUC 19 vector was digested with appropriate restriction enzymes, ligated with the amplified DNA fragments, and then applied for transforming E. coli DH5a. The plasmid DNAs multiplied in the cells were extracted, and purified.

\section{Intracellular ROS content}

IM303 (14) and MM294 cells carrying pUC 19 vector or constructed plasmid were cultivated under indicated conditions, and an aliquot of culture broth was withdrawn from the test tube to determine the intracellular ROS content at $\mathrm{OD}_{660}=0.5$ and $\mathrm{OD}_{660}=0.2$. ROS content was quantified with 5-(and-6)-chloromethyl-2',7'dichlorofluorescin diacetate (C-6827, Molecular Probes Inc., USA), and was expressed on a $\mathrm{H}_{2} \mathrm{O}_{2}$ equivalent basis (Krishnaiah et al., 2006). 


\section{RESULTS AND DISCUSSION}

The original population of SOD null mutant of E. coli, IM303, contained a trace of spontaneously derived variant cells, and these variant cells were permitted to survive, and prevail during the culture with the sublethal oxidative stress generated from light-irradiated $\mathrm{TiO}_{2}$. The growth profiles of the culture of a typically selected isolate, IM303 (14), with $\mathrm{TiO}_{2}$ and light, and without $\mathrm{TiO}_{2}$ and light were plotted as shown in Figure 2. The $\mu_{\mathrm{m}}$ and $\mathrm{t}_{\mathrm{t}}$ values were calculated with Gompertz equation as shown in Table 1. It is evident that the $\mu_{\mathrm{m}}$ value with $\mathrm{TiO}_{2}$ and light is approximately 2 times higher than that of the cells cultivated in the absence of $\mathrm{TiO}_{2}$ and light whereas the lag time lengths were appreciably distinct. To investigate the genomic-wide expression profiles in IM303 (14) cells cultivated under the oxidative stress, while comparing with those under the normal culture condition, DNA microarray analysis was performed. In total, 25 genes were up-regulated at a significant expression level by the oxidative stress and three genes, yfiD, yggB and $y g g E$, were selected for further research because literatures dealing with these genes were very few and the gene products may function to protect bacterial cells from oxidative stress. Based on the results mentioned above, three plasmids, pYFD, pYGB and pYGE, containing yfiD, $y g g B$ and $y g g E$, respectively, were constructed, and transformed into $E$. coli cells. Antioxidant functions of the genes were investigated in the cultures of the transformants under various oxidative conditions.

Table 1: Maximum specific growth rate $\mu_{\mathrm{m}}\left(\mathrm{h}^{-1}\right)$ and lag time $t_{L}(h)$ under various conditions

\begin{tabular}{ccc}
\hline Condition & $\boldsymbol{\mu}_{\mathrm{m}}$ & $\mathbf{t}_{\mathrm{L}}$ \\
\hline Without $\mathrm{TiO}_{2}$ and light & 0.20 & 7.8 \\
With $\mathrm{TiO}_{2}$ and light & 0.39 & 19.7 \\
\hline
\end{tabular}

The standard deviation was less than 0.01 per $\mathrm{h}$

In general, cell growth rate can be linked to an intracellular ROS level, which may be fluctuated in response to oxidative stress against cells, although several antioxidant systems exist in living cells to avoid cellular damages caused by oxidative stress (Storz and imlay, 1999, Kim et al., 2004a). As shown in Figure 3, the ROS contents in yfiD, yggB and yggE are less than the control pUC19 at both pre-exponential OD levels $\left(\mathrm{OD}_{660}=0.5\right.$ and $\left.\mathrm{OD}_{660}=0.2\right)$, despite ROS level in the transformant carrying $y g g E$ gene was suppressed significantly compared to $y f i D$ and $y g g B$, and further researches were focused on elucidating the functions of yggE gene product.

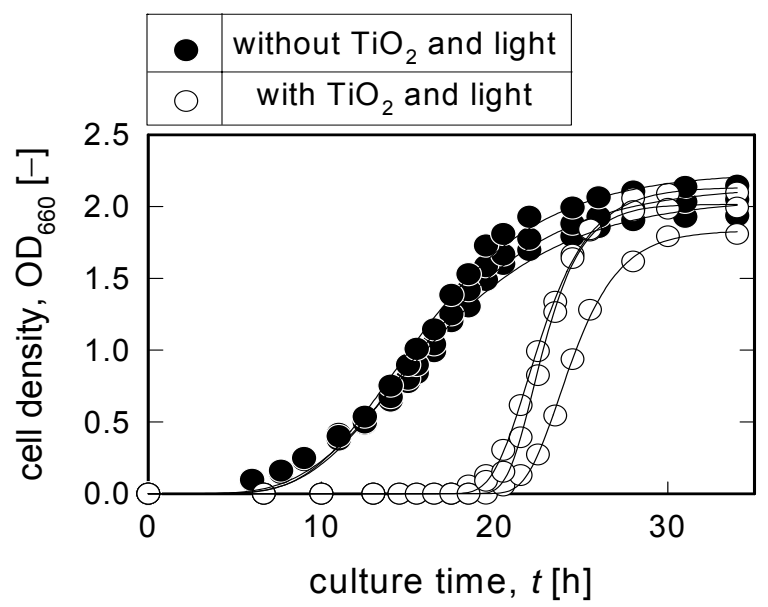

Figure 2: Growth profiles of typical isolate, IM303 (14) cells grown in the modified M9 medium with and without $\mathrm{TiO}_{2}$ and light. The solid lines were drawn by fitting the data to the modified Gompertz equation

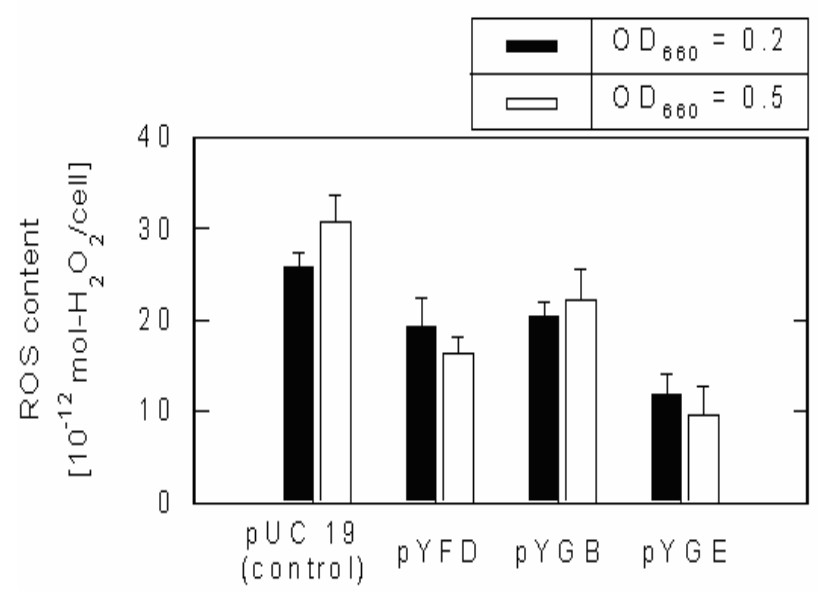

Figure 3: ROS contents in SOD-deficient mutant of $E$. coli, IM303 (I4) cells, carrying pUC 19, pYFD, pYGB and pYGE, measured at $\mathrm{OD}_{660}=0.2$ and 0.5 . The cells were grown in the modified $\mathrm{M} 9$ medium without $\mathrm{TiO}_{2}$ and light

Transformant carrying pYGE showed much higher $\mu_{\mathrm{m}}$ value than that of control cells carrying pUC 19 (Duduku et al., 2006). For investigating the biological function of $y g g E$ gene under different stress conditions, 
MM294 cells transformed by pYGE was cultivated in the medium with $10 \mu \mathrm{mol} / \mathrm{L} P Q$. As shown in Figure 4(A), the $\mu_{\mathrm{m}}$ value of the transformant was about 5 times higher than that of the control cells. In the case of ROS contents in the transformants carrying pUC 19 and pYGE, the latter cells showed significantly lower ROS levels than that of its counterpart as shown in Figure $4(B)$ at $O D_{660}=$ 0.5 and $\mathrm{OD}_{660}=0.2$.
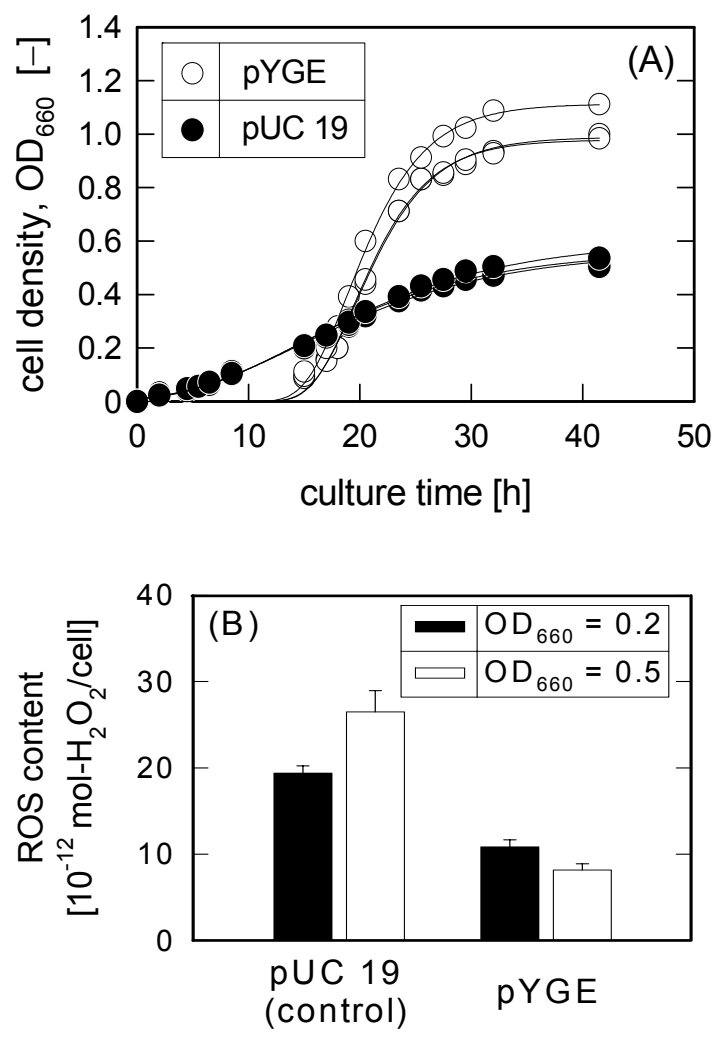

Figure 4: Growth profiles and ROS content in wild-type E. coli cells carrying pUC 19 and pYGE cultivated with $10 \mu \mathrm{mol} / \mathrm{L} P Q$. (A) Growth profiles of MM294 cells. The solid lines were drawn by fitting the data to the modified Gompertz equation. (B) ROS contents in MM294 cells

To investigate the effect of $y g g E$ gene product on culture property, cultivation of E. coli IM303 (14) carrying the plasmid pYGE or pUC 19 in a bioreactor was performed under aerobic conditions (Duduku et al., 2006). During the cultivation, DO concentration was kept at the value ranging from 6.0 to $6.8 \mathrm{ppm}$ as shown in Figure 5(A), which seems to be sufficient to provide stress to the SOD-deficient cells, and the sensitivity of oxygen was reported (Semchyshyn et al., 2005). Figure $5(\mathrm{~B})$ shows the growth profiles of E. coli IM303 (14) cells carrying $\mathrm{PYGE}$ and pUC 19, and time profiles of glucose concentration in medium. The cell density remained unchanged for both the transformants. DO concentration for pYGE was found to be higher than the pUC19 while the glucose concentration of pUC 19 is higher than pYGE. IM303 (14) cells carrying pYGE were remarkably enhanced in their growth activity when compared with that of the control cells carrying pUC 19. Furthermore, the dry cell weight obtained at the stationary phase of IM303 (14) cells carrying pYGE was significantly higher than that of the control cells carrying pUC19 in spite of no significant difference in glucose consumption.
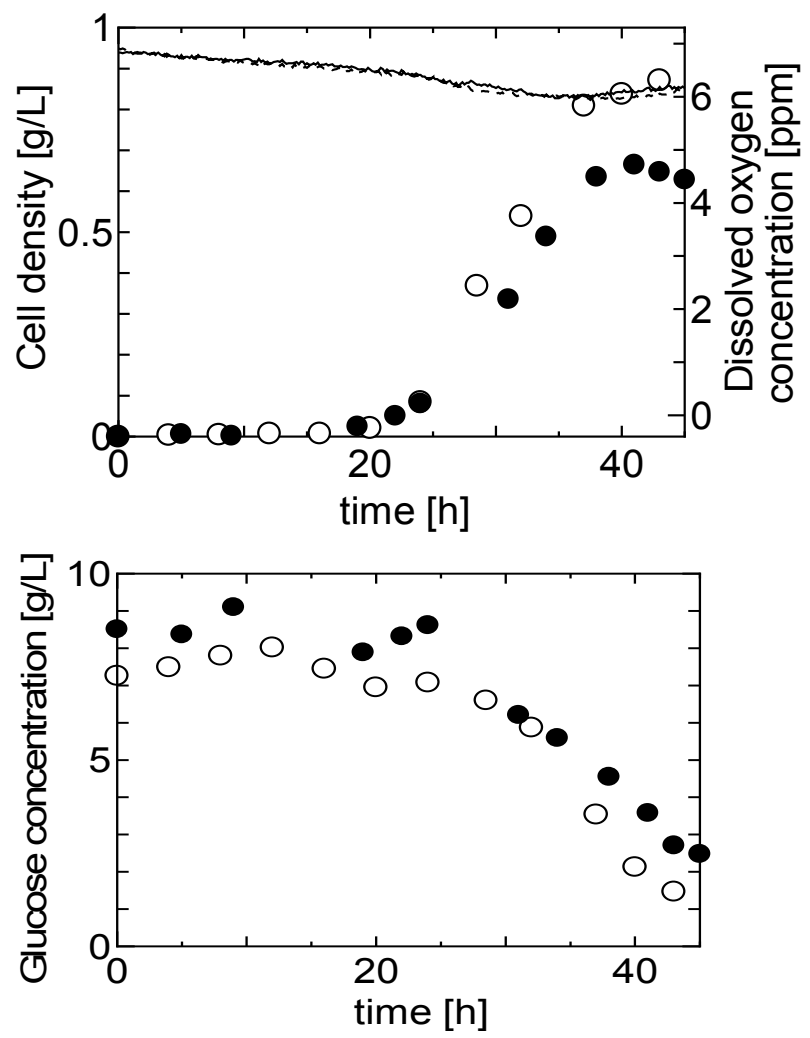

Figure 5: (A) Growth and dissolved oxygen concentration profiles of IM 303 (I4) cells carrying pYGE and pUC 19. (B) Time profiles of glucose concentration in the medium

\section{CONCLUSIONS}

Total gene expression profile in SOD-deficient E. coli cells was analyzed to understand their genotypic 
features. Three genes were selected for investigating their antioxidant functions. ROS level in the transformant carrying $y g g E$ gene was suppressed significantly compared to yfiD and yggB under the photoexcited $\mathrm{TiO}_{2}$ oxidative stress. IM303 (14) cells carrying pYGE were remarkably enhanced in their growth activity when compared with that of the control cells carrying pUC 19. Outcomes of the present work will make notable contribution to antioxidant defense systems in the fields of environmental and industrial approaches.

\section{REFERENCES}

Armon, R., Laot, N. and Narkis, N. (1998). Photocatalytic inactivation of different bacteria and bacteriophages in drinking water at different $\mathrm{TiO}_{2}$ concentrations with or without exposure to $\mathrm{O}_{2}$. Journal of Advanced Oxidation Technologies 3: 145 $-150$.

Bekbolet, M. (1997). Photocatalytic bactericidal activity of $\mathrm{TiO}_{2}$ in aqueous suspensions of E. coli. Water Sci. Technol. 35: 95 - 100.

Carlioz, A. and Touati, D. (1986). Isolation of superoxide dismutase mutants in Escherichia coli: Is superoxide dismutase necessary for aerobic life? EMBO Journal 5: $623-630$.

Canghai, Lu., Renee Albano, C., William, E.B. and Govind, Rao. (2005). Quantitative and kinetic study of oxidative stress regulons using green fluorescent protein. Biotechnology and Bioengineering 89: 574 587.

Farr, S.B. and Kogoma, T. (1991). Oxidative stress responses in Escherichia coli and Salmonella typhimurium. Microbiological Reviews 55: 561 - 585.

Gort, A.S. and Imlay, J. A. (1998). Balance between endogeneous superoxide stress and antioxidant defense. Journal of Bacteriology 180: 1402 - 1410.

Halliwell, B. Gutteridge, J.M.C. (1989). In: free radicals in biology and medicine, New York: Oxford University Press, 481 - 494.

Inaoka, T. Matsumura, Y. and Tsuchido, T. (1998). Molecular cloning and nucleotide sequence of the superoxide dismutase-deficient mutant of Escherichia coli. Journal of Bacteriology 174: 953 961.

Ireland, J.C., Klostermann, P., Rice, E.W. and Clark, L. (1993). Inactivation of Escherichia coli by titanium dioxide photocatalytic oxidation. Applied and Environmental Microbiology 59: 1668 - 1670.

Kim, S.Y., Nishioka, M., Hayashi, S., Honda, H., Kobayashi, T. and Taya, M. (2005). The gene yggE functions in restoring physiological defects of Escherichia coli cultivated under oxidative stress conditions. Applied and Environmental Microbiology 71(5): 2762 - 2765.
Kim, S.Y., Nishioka, M. and Taya, M. (2004). Promoted proliferation of SOD -deficient mutant of Escherichia coli under oxidative stress induced by photoexicited TiO2. FEMS Microbiology Letters 236: 109 - 114.

Kim, S.Y., Taguchi, T., Nishioka, M. and Taya, M. (2004a). Quantitative assessment of DNA damage accompanied with no substantial loss in its molecular weight during exposure to oxidative stress. Biochemical Engineering Journal 22: 81 - 87.

Kren, B., Parsell, D. and Fuchs, J.A. (1988). Isolationand characterization of an Escherichia coli K-12 mutant deficient in glutaredoxin. Journal of Bacteriology 170: 308 - 315.

Krishnaiah, D., Bono, A. and Sarbatly, R. (2006). Cultural characteristics of recombinant Escherichia coli cells carrying a novel antioxidant gene. Malaysian Journal of Microbiology 2: 10 - 14.

Pomposiello, P.J. and Demple, B. (2000). Encyclopedia of Microbiology 2nd Ed, Vol 3, Academic Press, New York. 78 - 84.

Semchyshyn, M., Lushchak, V. and Storey, K. (2005). Possible reasons for difference in sensitivity to oxygen of two Escherichia coli strains. Biochemistry (Moscow). 70: 424 - 431.

Storz, G. and Imlay, J.A. (1999). Oxidative stress. Current Opinion in Microbiology 2: 188 - 194.

Touati, D. (2002). Investigating phenotypes resulting from a lack of superoxide dismutase in bacterial null mutants. Methods in Enzymology. 349: 145 - 154.

Zwietering, M.H. Joongenburger, L. Rombouts, F.M. and van't Riet, K. (1990). Modeling of the bacterial growth curve. Applied and Environmental and Microbiology 56: 1875 - 1881. 